\title{
Optic Neuritis As a Presenting Symptom of Post-COVID-19 Multisystem Inflammatory Syndrome in Children (MIS-C)
}

\author{
Amrita Sinha ${ }^{1}$ [D $\cdot$ Deepak Dwivedi ${ }^{1} \cdot$ Anamika Dwivedi $^{1}$ - N. Bajaj ${ }^{1}$
}

Received: 9 July 2021 / Accepted: 28 July 2021 / Published online: 11 August 2021

(c) Dr. K C Chaudhuri Foundation 2021

To the Editor: Association of optic neuritis with various viral infections is well known [1] but it has not yet been described in multisystem inflammatory syndrome in children (MIS-C) associated with COVID-19. We describe a case of MIS-C in a child who presented with sudden loss of vision.

A 13-y-old male presented with $10 \mathrm{~d}$ of fever, conjunctival congestion, malaise, abdominal pain, itchy rash starting on the face and spreading to other areas, with blurring of vision which was sudden, painless, and progressive.

Physical exam showed diffuse, nonblanching erythematous rash on the bilateral upper/lower extremities and face. An elevated WBC count with left shift and lymphopenia, thrombocytopenia, increased CRP, and D-dimer. He was PCR-negative but had an increased antibody titer (33.96) for COVID-19. MRI brain, orbits, and other serological tests (anti-MOG antibody, anti-NMDA antibody) were normal. Hence, diagnosis of MIS-C was made.

On ocular examination, his visual acuity was $3 / 60$ (OU). Pupils were normal in size but sluggishly reacting to light. On fundus examination, both optic discs were hyperaemic, elevated with blurring of margins and obliteration of optic cup. Optical coherence tomogram (OCT) examination of BE optic disc was consistent with the findings of disc edema. A clinical diagnosis of bilateral optic neuritis was made.

The patient was managed with IV antibiotics, anticoagulant, IVIG, and methyl prednisone. Patient slowly improved, disc edema subsided, and near total restoration of visual functions with improvement in visual acuity BE was observed.

Viral infections play a role in increased permeability of the BBB allowing antibodies to cross it, can activate the immune system through molecular mimicry, and promote autoimmune responses through bystander activation [2]. COVID-19 has been implicated in several autoimmune diseases including GBS [3]. Murine coronaviruses are known to affect the central nervous system [4]. Therefore, there may be a causal link, in our patient, between SARS-CoV-2 and the subsequent development of optic neuritis.

\section{Declarations}

Conflict of Interest None.

\section{References}

1. Fernández Alcalde C, Granados Fernández M, Nieves Moreno M, Calvo Rey C, Falces Romero I, Noval MS. COVID-19 ocular findings in children: a case series. World J Pediatr WJP. 2021;17:329-34.

2. Ruijter NS de, Kramer G, Gons RAR, Hengstman GJD. Neuromyelitis optica spectrum disorder after presumed coronavirus (COVID-19) infection: A case report. Mult Scler Relat Disord. 2020;46:102474.

3. Toscano G, Palmerini F, Ravaglia S, et al. Guillain-Barré syndrome associated with SARS-CoV-2. N Engl J Med. 2020;382:2574-6.

4. Seah I, Agrawal R. Can the Coronavirus disease 2019 (COVID19) affect the Eyes? a review of Coronaviruses and ocular implications in humans and animals. Ocul Immunol Inflamm. 2020;28:391-5.

Publisher's Note Springer Nature remains neutral with regard to jurisdictional claims in published maps and institutional affiliations.

Amrita Sinha

Amritasinha1301@gmail.com

1 Department of Pediatrics, Shyam Shah Medical College, Rewa, Madhya Pradesh 486001, India 